\title{
Perspective: Informed consent in emergency care research: An oxymoron?
}

Jeremy S Furyk, Luke D Lawton, Joseph YS Ting, David McD Taylor for the Australasian College of Emergency Medicine - Clinical Trials Group (ACEM CTG)

Dr Jeremy S Furyk MBBM MPH\&TM MSc FACEM

Department of Emergency Medicine, The Townsville Hospital, 100 Angus Smith

Drive, Douglas, Townsville, Queensland, Australia 4814

College of Public Health and Medical and Veterinary Sciences, James Cook

University, Queensland, Australia

College of Medicine and Dentistry, James Cook University, Queensland, Australia

Phone: +61 744331111 Facsimile +61 744332901

Jeremy.Furyk@health.qld.gov.au

Twitter: @JeremyFuryk

Dr Luke D Lawton BAppSc MBBS (Hons) MPH

Department of Emergency Medicine, The Townsville Hospital, 100 Angus Smith

Drive, Douglas, Townsville, Queensland, Australia 4814

College of Medicine and Dentistry, James Cook University, Queensland, Australia

Joseph YS Ting

Emergency Department, Mater Health Service, Brisbane, Queensland, Australia School of Public Health and Social Work, Queensland University of Technology, Brisbane, Queensland, Australia

Professor David McD Taylor MBBS MPH DRCOG MD FACEM

Director of Emergency Medicine Research

Chair, Austin Health, Human Research Ethics Committee

Austin Hospital, Studley Road, Heidelberg, Victoria, Australia 3084

Department of Medicine, University of Melbourne, Parkville, Victoria, Australia

Contribution: All authors assisted with the conception of the article, as well as drafting and editing of the manuscript.

\section{Word Count: 1555}

This is the author manuscript accepted for publication and has undergone full peer review but has not been through the copyediting, typesetting, pagination and proofreading process, which may lead to differences between this version and the Version of Record. Please cite this article as doi: $10.1111 / 1742-6723.12642$

This article is protected by copyright. All rights reserved. 


\begin{abstract}
:
Emergency care needs to be underpinned by the highest quality evidence. However, research involving critically ill patients in the emergency setting, has unique ethical, logistical and regulatory issues. Informed consent is a well-established principle in conventional research. In this article, we discuss informed consent as it pertains to the difficulties of research in the emergency setting. Alternatives to informed consent are discussed. Human research ethics committees require a greater understanding of consent issues in emergency care research for Australia to remain competitive internationally.
\end{abstract}

\title{
Key Words:
}

Emergency medicine, informed consent, ethics, research

This article is protected by copyright. All rights reserved. 


\section{Introduction:}

Emergency care research differs from conventional models. Every day thousands of patients in Australasia present to pre-hospital services or emergency departments (ED) and expect care underpinned by the highest quality evidence. Regrettably, many emergency therapies lack a solid evidentiary base. Many well-intentioned interventions have been found to be harmful after proper scientific study, a phenomenon termed medical reversal ${ }^{1,2}$. Clinical trials are thus needed to delineate truly "best practice".

Informed consent is a well-established principle in conventional research ${ }^{3}$. However, its role in the emergency setting deserves discussion contextually within the complex ethical, logistic and regulatory issues unique to research in critically ill patients. While many factors make research difficult in the emergency setting, there is confusion around the requirement for informed consent and the ethical implications for research when informed consent is impossible.

\section{What is informed consent?}

This article is protected by copyright. All rights reserved. 
Informed consent is the exercise of a voluntary choice to participate in research based on the provision and subsequent comprehension of information about the purpose, methods, demands, risks, inconveniences, discomforts and possible outcomes of the research. ${ }^{3}$ Importantly, a signature on a consent form does not equate to informed consent ${ }^{4}$, and obtaining informed consent does not guarantee intrinsically ethical research ${ }^{5}$.

In Australia valid consent requires three elements: the capacity for decision making; a free and voluntary process; and adequate disclosure regarding the act performed. The absence of any element may 'transform the treatment into a potential assault' 6 .

Valid informed consent can often be obtained prior to enrolment in emergency care research. However, ED staff need to be trained in the principles of Good Clinical Practice, provide comprehensive information, allow sufficient time for consideration without coercion, and not prejudice clinical care after refusal.

\section{Is informed consent possible in emergency care research?}

Many important, unresolved clinical questions involve time-critical interventions in critically ill or injured patients who lack mental capacity because of their illness. In 
these circumstances, informed consent may not be feasible. Even if the patient retains some degree of capacity, the informed consent process may perceptually or materially impede the ability to treat the patient expeditiously. Further, the potential for coercion exists with all ED patients. Many present to the ED at a time of crisis and may be anxious, sick, in pain, disoriented or otherwise more vulnerable. Care must be taken to ensure that the consent process does not equate to exploitation.

Thus, where possible, informed consent should be sought but appropriate consideration should be given to the unique emergency setting so that consent can represent what is intended. Given both the issues unqiue to emergency medicine research and the complexity of consent arrangements which may need to be considered, it may be that special training or accreditation is warranted for HRECs considering emergency care research proposals.

\section{Alternatives to Informed consent.}

Alternatives to prospective informed consent include proxy consent, a waiver from individual participant consent, and retrospective or deferred consent. In Australia, decisions about persons who lack capacity to make decisions for themselves (either 
temporarily or permanently) are facilitated by jurisdictional guardianship legislation (State and Territory). Each jurisdiction has enacted its own legislation with the common law playing a limited role ${ }^{6}$. Confusion among emergency physicians, ethicists, legal advisors and Human Research Ethics Committees (HREC) around differences between the guardianship requirements of each jurisdiction and terminology in documents suggests the special circumstances of emergency, prehospital and critical care research were not adequately considered when the legislation was drafted.

The NHRMC specifies conditions for patients who cannot consent for themselves but these are subject to higher regulatory authority in all Australian jurisdictions $s^{3,7}$. Accordingly, large multi-national clinical trials have frequently been conducted with varying consent procedures in differing jurisdictions. This may itself be unethical and has the potential to bias the results.

\section{Proxy Consent}

Seeking a surrogate decision maker (proxy consent) is an option when the participant is incapable of providing informed consent. Indeed, this is the usual procedure for invasive medical therapy when a patient otherwise lacks capacity. 
However, there is evidence that the proxy may demonstrate poor agreement with the wishes of the participant ${ }^{8}$. Also, proxy consent may not be available in the timely manner necessary for interventions with a narrow therapeutic window ${ }^{3}$. In the CRASH trial of corticosteroids for severe head injuries, proxy consent was associated with a delay to treatment of 1.2 hours ${ }^{9}$. Also, proxies may be too distressed to provide truly informed consent.

\section{Waiver of informed consent}

Waiver of informed consent is permissible in certain circumstances unless prohibited by law ${ }^{3}$. While this raises concerns about unethical practice and contravention of the individual's autonomy, it provides patients the opportunity to participate in research and fulfils the ethical principle of justice ${ }^{5}$. To qualify for waiver of consent, the NHMRC National Statement (section 2.3.6 (a)) requires that the research "carries no more than low risk" 3 and that the "only foreseeable risk is one of discomfort". This is of little relevance to patients who are critically unwell and is unsuited to the unique nature of emergency research, which often, by its nature, is "high risk". It might be more useful to apply a concept of appropriate "incremental risk" 10,11.

This article is protected by copyright. All rights reserved. 
When emergency care research requires the waiver of informed consent, the responsibility to protect participants falls with the rigorous human research ethics approval process. The HREC must balance waiving the patient's right to consent, the importance of undertaking the research and societal benefit, and the potential lost opportunity for the patient to participate. Committees familiar with the "conventional" model of medical research consent may not be cognisant of the specific issues of emergency research, again suggesting that emergency care research should be considered by HRECs with a comprehensive understanding of these issues. Not infrequently, the same trial is conducted with differing consent requirements among the participating institutions, even if operating under the same ethical guiding principles and legal framework.

\section{Deferred consent}

Deferred consent is used when it is not possible to obtain prospective informed consent from the participant or proxy. However, its undertaking requires strict adherence to the relevant legislative requirements. Consent is obtained from the participant (or proxy) as soon as is feasible after the intervention. It allows the participant to remain in the trial for use of collected data and follow up.

This article is protected by copyright. All rights reserved. 
The advantages of waiver and deferred consent include avoidance of selection bias by maximising recruitment and the inclusion of sicker patients. It allows the study intervention to be delivered rapidly in the emergency setting. Deferred consent may also reduce staff anxiety about implementing a trial protocol and allows less deviation from routine clinical care ${ }^{12}$.

\section{Other approaches}

Barren et $\mathrm{al}^{13}$ suggested an adaptation of informed consent procedures for the emergency setting by "eliminating some of the less essential elements". In this model, time pressures and patient vulnerability exist but patients may still have capacity to consent or refuse. Clarification of which elements of consent should be included or excluded is required.

The controversial Zelen trial design ${ }^{14}$ has been proposed when comparisons are made to an accepted standard of care. Randomisation occurs before consent is obtained and generally consent is only sought in the intervention arm. Arguably, this reduces unnecessary anxiety among those allocated to standard treatments and improves recruitment rates.

This article is protected by copyright. All rights reserved. 


\section{Research into alternatives to informed consent}

Research into alternatives to informed consent is lacking. In a hypothetical trial of trauma management, the majority of patients were found not able to consent ${ }^{15}$. Experience in large trials using different consent procedures has demonstrated that these factors do influence both recruitment rates and times to intervention delivery. ${ }^{9}$ This has implications for trial success, patient outcomes and therefore the ethical acceptability of the research. Reassuringly though, the available evidence suggests that approximately $70 \%$ of patients support the idea of research without informed consent in some circumstances 16,17 . The perceived risk of the intervention seems to be related to support for research without consent. For researchers, it is difficult to understand a patient's willingness to accept an unproven clinical care intervention recommended by their doctor, compared with some reluctance when the patient is told that their data will be collected as part of a study or that they are to be randomised ${ }^{11,16}$. Concerns about the process of randomisation are common, with perhaps little understanding of its scientific importance ${ }^{18}$. In reality, more rigorous protections are in place as part of clinical trial protocols rather than the various whims of individual practitioners during routine care.

\section{Conclusion}

This article is protected by copyright. All rights reserved. 
Informed consent in its truest form is difficult to obtain in the emergency setting and there is a need for greater clarity regarding appropriate alternatives, particularly for delineation of an acceptable standard. It is important to understand the factors that influence patient and public trust in medical research. Human research ethics committees are encouraged to consider the ethical acceptability of differing consent procedures as a way of expediting and standardising ethical review and protecting trial participants, while satisfying the community expectation that emergency care is evidence-based. Specific accreditation of HRECs with expertise in emergency care research is recommended in order to standardise consent procedures across jurisdictions. HRECS require a greater understanding of consent issues in emergency care research for Australia to remain competitive internationally. Australia needs consistency in its approach to informed consent in emergency research in order to facilitate participation in large, well-conducted trials designed to improve patient care.

Acknowledgements: The authors are grateful to Dr Nikola Stepanov for advice on early drafts of the manuscript

\section{References}

This article is protected by copyright. All rights reserved. 
1. Fatovich DM. Medical reversal: What are you doing wrong for your patient today? Emergency medicine Australasia : EMA. 2013 Feb;25(1):1-3. PubMed PMID: 23379445.

2. Prasad V, Gall V, Cifu A. The frequency of medical reversal. Archives of internal medicine. 2011;171:1675-6.

3. NHMRC. National Statement on Ethical Conduct in Human Research (2007) Updated May 2015. Canberra: National Health and Medical Research Council; 2007. 4. Coats TJ. Ethical and practical issues in trauma care research. The British journal of surgery. 2012 Jan;99 Suppl 1:6-7. PubMed PMID: 22441848. Epub 2012/03/28. eng.

5. Lecouturier J, Rodgers H, Ford GA, Rapley T, Stobbart L, Louw SJ, et al. Clinical research without consent in adults in the emergency setting: a review of patient and public views. BMC medical ethics. 2008;9:9. PubMed PMID: 18445261. Pubmed Central PMCID: PMC2390563. Epub 2008/05/01. eng.

6. White B, McDonald F, Willmontt L. Health Law in Australia. Australia: Thomson Reuters; 2014.

7. Turner E. Substitute descision-making for participation in medical research. Australian Health Law Bulletin. 2015 May:66-70.

8. Booth MG. Informed consent in emergency research: a contradiction in terms. Science and engineering ethics. 2007 Sep;13(3):351-9. PubMed PMID: 18210228. Epub 2008/01/23. eng.

9. Roberts I, Prieto-Merino D, Shakur H, Chalmers I, Nicholl J. Effect of consent rituals on mortality in emergency care research. Lancet. 2011 Mar 26;377(9771):1071-2. PubMed PMID: 21439634. Epub 2011/03/29. eng. 10. Biros M, Lewis RJ, Olson C, Runge JW, Cummins R, Fost N. Informed consent in emergency research. JAMA. 1995;273(16):1283-87.

11. Gray JD. The problem of consent in emergency medicine research. Cjem. 2001 Jul;3(3):213-8. PubMed PMID: 17610787. Epub 2007/07/06. eng. 12. Ripley E, Ramsey C, Prorock-Ernest A, Foco R, Luckett Jr S, Ornato JP. EMS Providers and Exception From Informed Consent Research: Benefits, Ethics, and Community Consultation. Prehosp Emerg care. 2012;16(4):425-33.

13. Baren JM, Fish SS. Resuscitation research involving vulnerable populations: are additional protections needed for emergency exception from informed consent? Academic emergency medicine : official journal of the Society for Academic Emergency Medicine. 2005 Nov;12(11):1071-7. PubMed PMID: 16264077. Epub 2005/11/03. eng.

14. Zelen M. A New Design for Randomized Clinical Trials. N Engl J Med. 1979;300:1242-5.

15. Dutton RP, Stansbury LG, Hemlock B, Hess JR, Scalea TM. Impediments to obtaining informed consent for clinical research in trauma patients. The Journal of 
trauma. 2008 Apr;64(4):1106-12. PubMed PMID: 18404082. Epub 2008/04/12. eng.

16. Abboud PA, Heard K, Al-Marshad AA, Lowenstein SR. What determines whether patients are willing to participate in resuscitation studies requiring exception from informed consent? Journal of medical ethics. 2006 Aug;32(8):46872. PubMed PMID: 16877627. Pubmed Central PMCID: PMC2563379. Epub 2006/08/01. eng.

17. Eltorki M, Uleryk E, Freedman SB. Waiver of informed consent in pediatric resuscitation research: a systematic review. Academic emergency medicine : official journal of the Society for Academic Emergency Medicine. 2013 Aug;20(8):822-34. PubMed PMID: 24033626. Epub 2013/09/17. eng. 18. Dickert N, Kass N. Patients' perceptions of research in emergency settings: a study of survivors of Sudden Cardiac Death. Soc Sci Med. 2009;68(1):183-91.

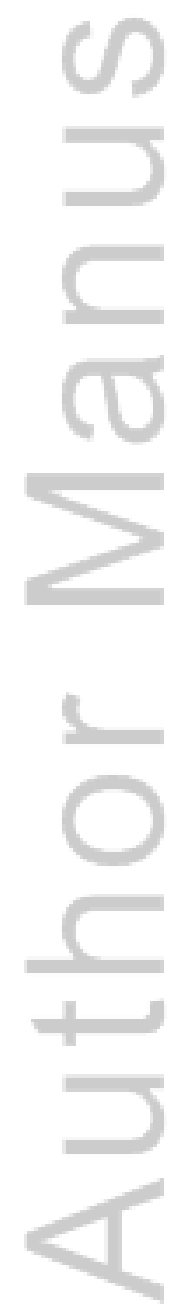

This article is protected by copyright. All rights reserved. 


\section{University Library}

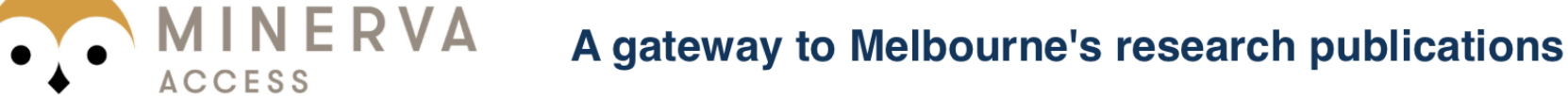

Minerva Access is the Institutional Repository of The University of Melbourne

Author/s:

Furyk, JS;Lawton, LD;Ting, JYS;Taylor, DM

Title:

Informed consent in emergency care research: An oxymoron?

Date:

2017-02-01

Citation:

Furyk, J. S., Lawton, L. D., Ting, J. Y. S. \& Taylor, D. M. (2017). Informed consent in emergency care research: An oxymoron?. EMERGENCY MEDICINE AUSTRALASIA, 29 (1), pp.110-112. https://doi.org/10.1111/1742-6723.12642.

Persistent Link:

http://hdl.handle.net/11343/291557 